\title{
Chaos-Induced Pulse Trains in the Ionization of Hydrogen
}

\author{
K. A. Mitchell \\ William \& Mary \\ J. P. Handley \\ William \& Mary \\ B. Tighe \\ William \& Mary \\ A. Flower \\ William \& Mary \\ John B. Delos \\ William \& Mary, jbdelos@wm.edu
}

Follow this and additional works at: https://scholarworks.wm.edu/aspubs

Part of the Physics Commons

\section{Recommended Citation}

Mitchell, K. A.; Handley, J. P.; Tighe, B.; Flower, A.; and Delos, John B., Chaos-Induced Pulse Trains in the Ionization of Hydrogen (2004). Physical Review Letters, 92(7).

https://doi.org/10.1103/PhysRevLett.92.073001

This Article is brought to you for free and open access by the Arts and Sciences at W\&M ScholarWorks. It has been accepted for inclusion in Arts \& Sciences Articles by an authorized administrator of W\&M ScholarWorks. For more information, please contact scholarworks@wm.edu. 


\title{
Chaos-Induced Pulse Trains in the Ionization of Hydrogen
}

\author{
K. A. Mitchell, J. P. Handley, B. Tighe, A. Flower, and J. B. Delos \\ Department of Physics, College of William and Mary, Williamsburg, Virginia 23187-8795, USA
}

(Received 12 May 2003; published 19 February 2004)

\begin{abstract}
We predict that a hydrogen atom in parallel electric and magnetic fields, excited by a short laser pulse to an energy above the classical saddle, ionizes via a train of electron pulses. These pulses are a consequence of classical chaos induced by the magnetic field. We connect the structure of this pulse train (e.g., pulse size and spacing) to fractal structure in the classical dynamics. This structure displays a weak self-similarity, which we call "epistrophic self-similarity." We demonstrate how this selfsimilarity is reflected in the pulse train.
\end{abstract}

DOI: 10.1103/PhysRevLett.92.073001

In recent experiments, the ionization rate versus time was measured for a rubidium atom excited by a short (a few picoseconds) laser pulse in a constant applied electric field [1]. Ionization occurred via a train of electron pulses, rather than by exponential decay. This observation can be qualitatively explained by the following semiclassical analysis [2]. Photoabsorption promotes the valence electron from a low-energy bound state into an outgoing wave, which can be modeled semiclassically as an ensemble of trajectories propagating away from the atomic core in all directions and with a narrow range of energies. Some trajectories head directly downhill and are accelerated by the external field toward a detector, creating an initial prompt pulse of electrons. Other trajectories initially head uphill, are turned around by the field, and return to the core where they scatter in all directions. Some scattered trajectories head downhill, creating a second pulse of electrons. Subsequent rescattering events create additional pulses. Such results have also been obtained in quantum computations [2].

In this Letter, we predict that somewhat similar pulse trains should occur for a hydrogen atom in parallel electric and magnetic fields [Fig. 1(a)]. However, the mechanism for pulse creation is fundamentally different from that described above. Pulses are not created through core scattering (since there are no core electrons). Rather, the pulses result from classical chaos, which is induced by the magnetic field. The experimental measurement of such pulse trains would thus not only elucidate a fundamental ionization mechanism, but would also provide a convenient laboratory tool for studying chaotic transport and escape.

Hamiltonian and Poincaré surface of section. - The Hamiltonian of a hydrogenic electron in parallel fields may be written in scaled units and cylindrical coordinates $(\rho, z)$ as

$$
\begin{array}{r}
H\left(\rho, z, p_{\rho}, p_{z}\right)=\frac{1}{2}\left(p_{\rho}^{2}+p_{z}^{2}\right)+V(\rho, z) \equiv E, \\
V(\rho, z)=-\frac{1}{\sqrt{\rho^{2}+z^{2}}}+z+\frac{1}{8} B^{2} \rho^{2},
\end{array}
$$

PACS numbers: $32.80 . \mathrm{Rm}, 05.45 . \mathrm{Ac}, 05.45 . \mathrm{Df}, 32.80 . \mathrm{Fb}$

where the $z$ component of angular momentum is taken to be zero and where $E=\hat{E} \hat{F}^{-1 / 2}$ and $B=\hat{B} \hat{F}^{-3 / 4}$ are the scaled energy and the scaled magnetic field strength, respectively; $\hat{E}, \hat{F}$, and $\hat{B}$ are, respectively, the physical energy and the physical electric and magnetic field strengths (in atomic units). Excited electrons initially move radially outward from the proton in the Coulomb potential well (Fig. 2), and ionization occurs by exiting the well past the saddle point and proceeding to $z=-\infty$.

We convert the continuous-time dynamics in the fourdimensional phase space $\left(\rho, z, p_{\rho}, p_{z}\right)$ into a discrete-time Poincaré map on the two-dimensional Poincaré surface of section $\left(z, p_{z}\right)$, defined by the constraints $\rho=0, z<0$, and $H=E$. The Poincaré map $\left(z, p_{z}\right) \mapsto\left(z^{\prime}, p_{z}^{\prime}\right)$ is constructed in the following way. A point $(\rho=0, z)$ on the negative $z$ axis in Fig. 2 is launched with a given value of $p_{z}$, and with $p_{\rho}$ determined by $H=E$. This trajectory initially moves away from the $z$ axis but is eventually turned around by the potential. It subsequently returns to the negative $z$ axis, where we record the new point $\left(z^{\prime}, p_{z}^{\prime}\right)$. To eliminate the singular Coulomb behavior, we refer the map to new canonical coordinates $(q, p)$ on the surface of section [3]. Figure 3 shows a surface-of-section plot in these new coordinates.

Homoclinic tangles and phase space transport. - Near the saddle of the potential energy in Fig. 2 is an unstable periodic orbit through which all escaping trajectories must pass. The intersection of this orbit with the $z$ axis corresponds to an unstable fixed point (or $\mathrm{X}$ point) of the Poincaré map. This fixed point, $\mathbf{z}_{\mathrm{X}}$, is shown in Fig. 3, along with its stable $(S)$ and unstable $(\mathcal{U})$ manifolds (i.e., the sets of points that converge to $\mathbf{z}_{X}$ under forward and backward iterates, respectively.) These manifolds intersect in a complicated pattern, called a homoclinic tangle.

A tangle, as in Fig. 3, produces phase space transport and escape [5-8]. The stable and unstable manifolds intersect transversely at a point $\mathbf{P}_{0}$, thereby bounding a region of the plane which we call the complex. Points within the complex correspond to the neutral atom; if a trajectory maps outside the complex, it will subsequently progress to infinity, resulting in ionization. Additional 


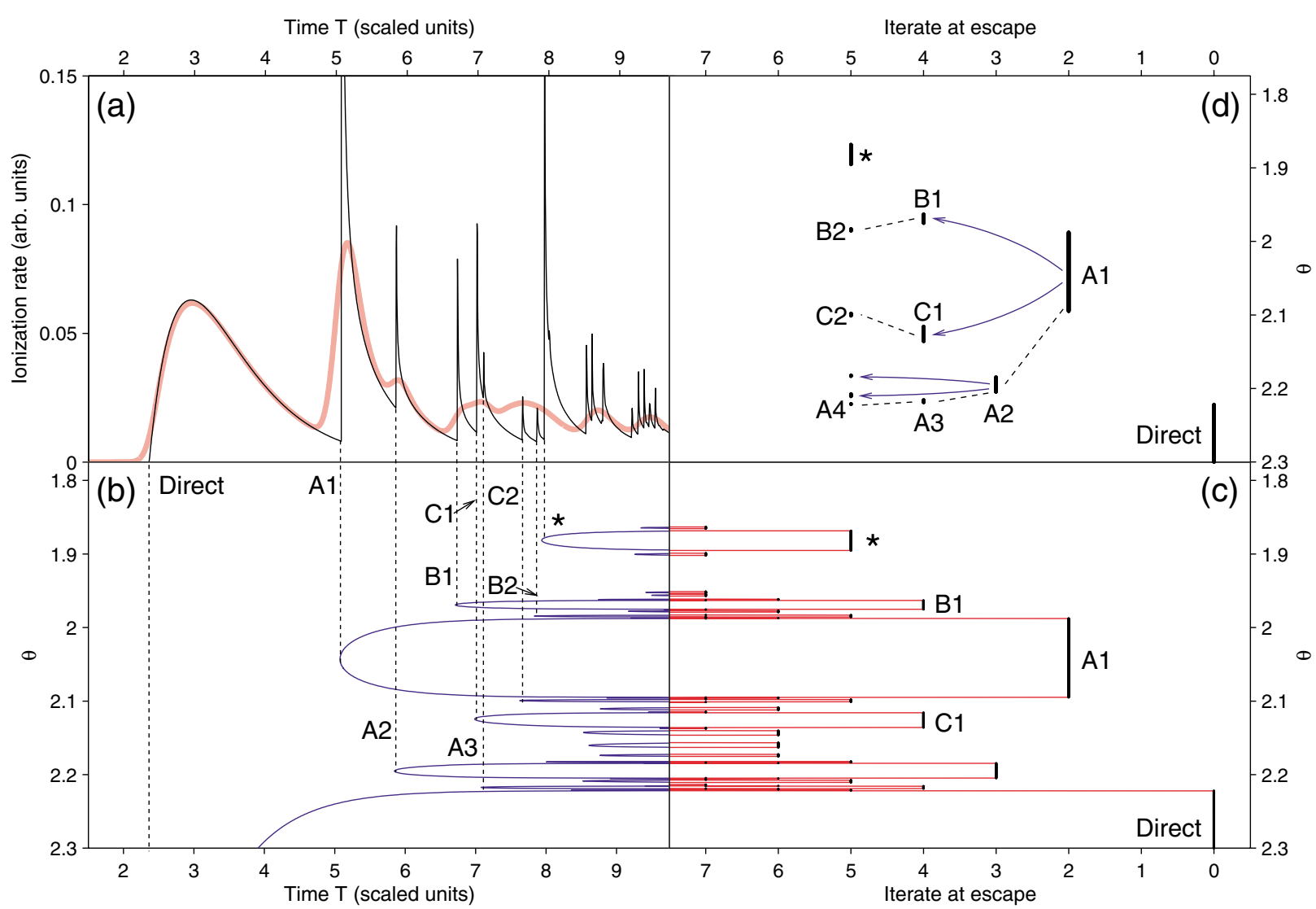

FIG. 1 (color online). (a) For hydrogen in parallel fields, with $B=4.5, E=-1.3$, the flux of electrons striking a detector (placed at $z=-4)$ is plotted as a function of time $T$ after the excitation of the atom. The thin dark line models the outgoing electron wave packet by an ensemble of trajectories with precise energy and start time. The thick shaded line uses an ensemble given by a minimum uncertainty Gaussian wave packet with central energy corresponding to effective quantum number $N \equiv(-2 \hat{E})^{-1 / 2}=$ 80. This implies physical parameters $\hat{B}=0.49 \mathrm{~T}, \hat{F}=19 \mathrm{~V} / \mathrm{cm}$, and one scaled unit of time $T$ equal to 52 ps. We used a wave packet of width $\Delta T=0.1$ scaled units $(5.2 \mathrm{ps})$. (b) The time it takes a trajectory to strike the detector is plotted as a function of the initial angle $\theta$ at which the trajectory is launched. The dashed lines connect icicles to their corresponding pulses in (a). The lowest icicle, having only its upper portion visible in (b), corresponds to the direct trajectories. (c) The number of iterates required to escape the complex is plotted as a function of $\theta$. Each escape segment corresponds to an icicle in (b). (d) The epistrophic structure of the first several escape segments is shown. The dashed lines connect segments within one epistrophe. The solid arrows show the creation of new epistrophes according to the epistrophe start rule. The asterisk denotes a strophe segment which does not fit into the pattern of epistrophes.

intersections $\mathbf{P}_{n}$ and $\mathbf{Q}_{n}$ of the stable and unstable manifolds define regions $E_{n}$ (escape lobes) and $C_{n}$ (capture lobes $),-\infty<n<\infty$, in the phase plane. For example, the segments of $S$ and $\mathcal{U}$ that join $\mathbf{P}_{0}$ to $\mathbf{Q}_{0}$ in Fig. 3 bound the lobe $E_{0}$. Under the Poincaré map, $E_{n} \mapsto E_{n+1}, C_{n} \mapsto$ $C_{n+1}, \mathbf{P}_{n} \mapsto \mathbf{P}_{n+1}$, and $\mathbf{Q}_{n} \mapsto \mathbf{Q}_{n+1}$.

Transport in and out of the complex occurs via a "turnstile" [5]: The escape lobe $E_{-1}$, which is inside the complex, maps to $E_{0}$, which is outside the complex; similarly, the capture lobe $C_{0}$, outside the complex, maps to $C_{1}$, inside the complex. All points that escape in $n$ iterates lie in the lobe $E_{-n}$.

The structure of the tangle is characterized by two quantities, a local geometric quantity $\alpha$ and a global topological quantity $D$ : (1) The Liapunov factor $\alpha>1$ is the largest eigenvalue of the Poincare map, linearized about the fixed point $\mathbf{z}_{X}$; (2) the minimum delay time $D$ is the smallest $n$ such that $E_{-(n+1)}$ intersects $C_{0}$. For Fig. 3, $\alpha=5.71$ and $D=1$.

Electron escape. - We model the electron wave function, immediately after the absorption of a photon from the short laser pulse, by an ensemble of classical trajectories that begin at the nucleus and propagate radially outward in all directions with fixed energy $E$. This ensemble forms a line of initial conditions $\mathcal{L}_{0}$ in the Poincaré plane (Fig. 3) parametrized by the angle $0 \leq$ $\theta \leq \pi$ between the initial velocity vector and the positive $z$ axis. The density of points along $\mathcal{L}_{0}$ is given by the angular distribution $|\mathcal{Y}(\theta)|^{2} \sin \theta d \theta$ of the outgoing wave [9], which depends on the experimental excitation 


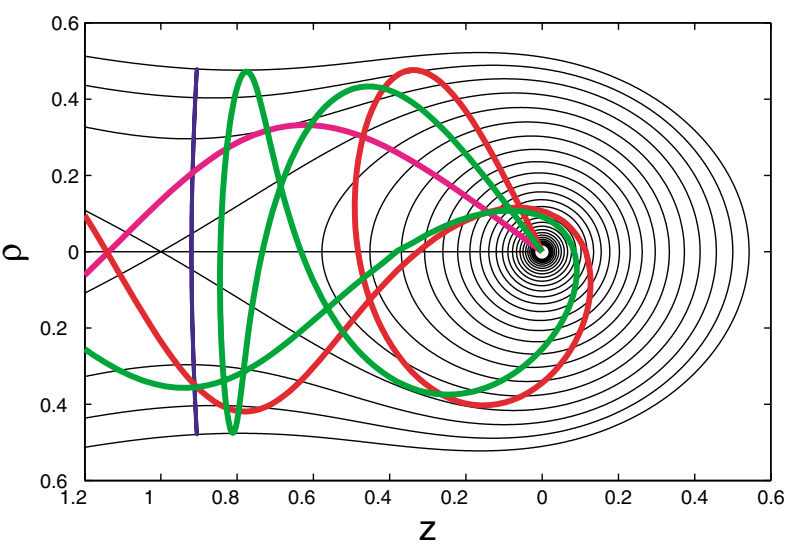

FIG. 2 (color online). A contour plot of potential energy is shown $(B=4.5 ; E=-1.3)$ with a prominent unstable periodic orbit (vertical curve) near the saddle point. Three ionizing trajectories are also plotted. The negative $z$ axis defines the surface of section.

scheme. The data in Fig. 1(a) are computed using a $p_{x}$ angular distribution; a different distribution would change the relative sizes of the pulses.

We measure how long it takes a trajectory to escape, as a function along $\mathcal{L}_{0}$, in two ways: (1) The continuousescape-time plot [Fig. 1(b)] shows the time $T$ it takes a trajectory to reach the detector. The plot is divided into an infinite number of smooth curves, which we call icicles following Ref. [10], that go to infinity at their end points. These infinite-time end points correspond to trajectories that converge to $\mathbf{z}_{\mathrm{X}}$; that is, they are intersections of $\mathcal{L}_{0}$ with the stable manifold. (2) The discrete-escape-time

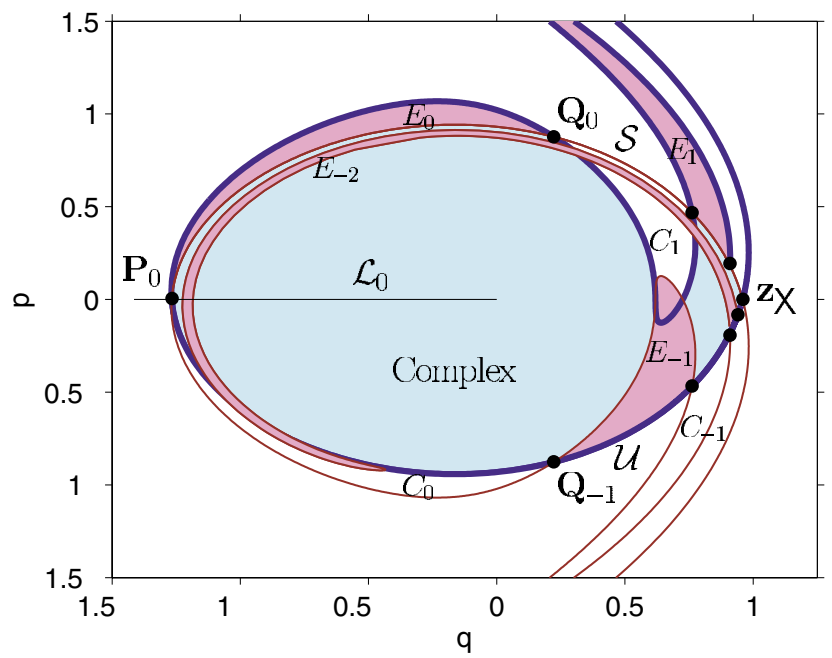

FIG. 3 (color online). A surface-of-section plot $(B=4.5$; $E=-1.3$ ) showing the unstable fixed point $\mathbf{z}_{X}$ with its homoclinic tangle of intersecting stable and unstable manifolds [the thin curve $(S)$ and the thick curve $(\mathcal{U})$, respectively]. The lobe $E_{-2}$ intersects the line of initial conditions $\mathcal{L}_{0}$, creating a segment that will escape (i.e., land in $E_{0}$ ) in two iterates. plot [Fig. 1(c)] shows the number of iterates of the Poincare map required for a trajectory to escape the complex. The discrete-time plot is a rectification of the continuous-time plot; each icicle is straightened into a single escape segment with constant iterate number. A segment that escapes in $n$ iterates is an intersection between the line of initial conditions and the lobe $E_{-n}$ of the tangle (Fig. 3) [11].

The ionization rate can be computed from the continuous-escape-time plot. Each branch in Fig. 1(b) contributes an amount $|\mathcal{Y}(\theta)|^{2} \sin \theta d \theta / d T$ to the total rate. Thus, each icicle produces a pulse of electrons; the integrated flux of the pulse is roughly proportional to the width of the icicle. Thus, to understand the structure of the electron pulse train, we study the structure of the escape-time plot.

Epistrophic structure of escape-time plots. - Throughout the escape-time plot [Fig. 1(c)] and on all scales there are regular sequences of escape segments, which we call epistrophes [12], having the following properties [7,8]: (1) An epistrophe is an infinite sequence of consecutive escape segments that converge monotonically upon the end point of another escape segment. (2) For any epistrophe, the convergence is geometric (in the asymptotic limit) with a rate equal to the Liapunov factor $\alpha$ of the fixed point $\mathbf{z}_{X}$. (3) The asymptotic tails of any two epistrophes differ only by a change of scale; the epistrophes are all asymptotically self-similar and similar to each other. (4) An epistrophe converges upon each end point of every escape segment [13], so the escape-time plot has structure within structure on all scales. The removal of escape segments on each iterate is analogous to the construction of the middle-third Cantor set, and the set of trajectories that never escape has an analogous fractal structure.

The epistrophe structure is illustrated in Fig. 1(d). The A epistrophe (of which the first four segments are shown) converges upon the upper end point of the direct segment. Similarly, the B and C epistrophes converge upon the upper and lower end points of the $A 1$ segment, respectively. The first segments (unlabeled) of the two epistrophes converging upon the upper and lower end points of the $A 2$ segment occur on the fifth iterate. The rapid decay of the escape segments in each epistrophe is a consequence of $\alpha=5.71$.

The beginnings of epistrophes are partially predictable. Topological arguments show that there must be a certain "minimal set" of escape segments [8]. The escape segments within this minimal set obey an "epistrophe start rule": An escape segment on the $n$th iterate spawns two new epistrophes on iterate $n+D+1$. This rule is seen in Fig. 1(d), for which $D+1=2$. The B and C epistrophes start at $n=4$, two iterates after the A1 segment at $n=2$. Similarly, the A2 segment $(n=3)$ spawns two new epistrophes at $n=5$.

Calculations show that there are also escape segments that do not belong to the minimal set. These segments do 
not fit the simple pattern predicted by the epistrophe start rule. Such a segment, which we call a strophe, is marked by an asterisk in Figs. 1(b)-1(d).

We use the term epistrophic self-similarity to describe the above situation: Throughout the escape-time plot and on all scales there are epistrophes; they are all asymptotically self-similar and each is similar to every other. However, there may also be strophe segments which occur on all scales and which, at long times, may come to dominate the regular epistrophe structure.

Epistrophic structure reflected in the pulse train. - To each escape segment there corresponds an icicle and, hence, an electron pulse. Thus, an epistrophe of escape segments produces an epistrophe of electron pulses, which decays exponentially with rate $\alpha$. In Figs. 1(a) and 1(b) we explicitly connect the first nine pulses to their corresponding icicles and label them according to the scheme in Fig. 1(d). The first pulse is the large direct pulse. The second, third, and sixth pulses are the first three pulses of the A epistrophe. Notice the rapid decay in pulse height. The time between successive pulses in an epistrophe is roughly equal to $T_{\mathrm{po}}=1.28$, the time it takes the periodic orbit (po) in Fig. 2 to leave and return to the $z$ axis.

The epistrophe start rule is also reflected in the pulse train. Since $D+1=2$, the two pulses $\mathrm{B} 1$ and $\mathrm{C} 1$, spawned by the $A 1$ pulse, occur roughly concurrently with the $A 3$ pulse, or roughly $2 T_{\text {po }}$ after the $A 1$ pulse. The B2 and $\mathrm{C} 2$ pulses, which are significantly smaller, follow roughly $T_{\mathrm{po}}$ later.

The next pulse in the train is associated with the strophe segment (marked by the asterisk). Notice that it is a large pulse compared to pulses nearby (in time). This is typical of the behavior we observe for the strophes, and numerical evidence indicates that the strophe pulses come to dominate at long times.

Quantum considerations. - What are the implications of our classical analysis for the true quantum dynamics? First, in light of the uncertainty principle, the ensemble of initial electrons cannot have both a sharp energy and a sharp starting time. We should therefore replace our initial ensemble with one covering a Plank cell in phase space; that is, we should average the thin line in Fig. 1(a) over a distribution of energies and starting times. The result of this averaging [using an initial minimum uncertainty Gaussian wave packet with average energy given by $N \equiv(-2 \hat{E})^{-1 / 2}=80$ ] is the thick line in Fig. 1(a). Although the sharp pulses are smoothed out, the pulse structure is still clearly visible [14]. For higher excitations $N$, the dynamics is more classical and the pulses are more clearly distinguished.

The second important quantum effect is interference; electrons traveling along two trajectories that arrive at the same point at the same time interfere constructively or destructively. We will explore interference in a future paper, but it will not change the pulsed character of the classical results. A third quantum effect is tunneling; our estimates indicate that it is negligible at short times.

Experimental observation of the chaotic pulse trains described here will shed light on chaotic behavior in open classical and quantum systems.

[1] G. M. Lankhuijzen and L. D. Noordam, Phys. Rev. Lett. 76, 1784 (1996); Opt. Commun. 129, 361 (1996).

[2] F. Robicheaux and J. Shaw, Phys. Rev. Lett. 77, 4154 (1996); Phys. Rev. A 56, 278 (1997).

[3] The coordinates $(q, p)$ used in Fig. 3 arise as follows. Following standard practice [4], we convert from cylindrical coordinates $(\rho, z)$ to parabolic coordinates $(u, v)$. On the Poincaré surface $\left\{\left(v, p_{v}\right) \mid u=0, H=E\right\}$, we define canonical polar coordinates $I=\left(p_{v}^{2}+v^{2}\right) / 2$, $\phi=-\tan ^{-1}\left(p_{v} / v\right)$. We then eliminate the inversion symmetry $\phi \mapsto \phi+\pi$ by defining $p=$ $-\sqrt{I} \sin 2 \phi=-2 p_{z} \sqrt{-z /\left(1+p_{z}^{2}\right)}, \quad q=\sqrt{I} \cos 2 \phi=$ $\left(1-p_{z}^{2}\right) \sqrt{-z /\left(1+p_{z}^{2}\right)}$.

[4] J. Gao and J. B. Delos, Phys. Rev. A 49, 869 (1994).

[5] R. S. MacKay, J. D. Meiss, and I. C. Percival, Physica (Amsterdam) 13D, 55 (1984); S. Wiggins, Chaotic Transport in Dynamical Systems (Springer-Verlag, New York, 1992).

[6] M. J. Davis and S. K. Gray, J. Chem. Phys. 84, 5389 (1986); M. J. Davis, J. Chem. Phys. 83, 1016 (1985); V. Rom-Kedar, Physica (Amsterdam) 43D, 229 (1990); Nonlinearity 7, 441 (1994).

[7] K. A. Mitchell, J. P. Handley, B. Tighe, S. K. Knudson, and J. B. Delos, Chaos 13, 880 (2003).

[8] K. A. Mitchell, J. P. Handley, S. K. Knudson, and J. B. Delos, Chaos 13, 892 (2003).

[9] M. L. Du and J. B. Delos, Phys. Rev. A 38, 1896 (1988); 38, 1913 (1988).

[10] L. Gottdiener, Mol. Phys. 29, 1585 (1975); A. Tiyapan and C. Jaffé, J. Chem. Phys. 99, 2765 (1993); 101, 10393 (1994); 103, 5499 (1995).

[11] Escape-time plots and related functions are standard tools in chaotic scattering theory. See, for example, Ref. [10] and the citations in Refs. [7,8].

[12] "Epistrophe" is a term from rhetoric meaning "a regular, repeated ending following variable beginnings."

[13] Strictly speaking, an epistrophe converges only upon an intersection of $\mathcal{L}_{0}$ with $S$; this includes all end points of escape segments interior to the complex.

[14] The duration of the laser pulse is important for resolving the electron pulses. A pulse that is too short has such a wide range in energy that the icicle plot [Fig. 1(b)] varies substantially over this range, washing out the pulses. Alternatively, a pulse that is too long washes out the pulses due to its large $\Delta T$ (at which point interference becomes more important). We used a pulse length of $5.2 \mathrm{ps}$ $(\Delta T=0.1)$ for the smoothing in Fig. 1(a). 